\title{
Research of Effect of Ergonomics on Athletic Shoes and Costume Design Project

\author{
Zhaozhong Jin ${ }^{1, a}$, Dong Chen ${ }^{2, b}$, Yang Yang ${ }^{3, c}$ \\ ${ }^{1}$ Hebei College of Industry and Technology, China \\ ${ }^{2,3}$ Hebei Chemical\&Pharmaceutical College, China
} \\ a50954099@qq.com, b419876845@qq.com, ${ }^{\mathrm{c}} 35949162 @ q q . c o m$
}

Keywords: ergonomics; athletic shoes and costume design project

\begin{abstract}
Different sport athletic events require different shoes and costume design requirements. Members such as gymnastics shoes and costume should be designed to ensure that the premise Athletes play freely, to display their graceful movements of the body; fencing shoes and costume design should first focus on protecting the body, followed by on-demand light and so on. This article is skipping example, combining ergonomics knowledge, studying on the effects of ergonomic on athletic shoes and costume design project .
\end{abstract}

\section{Introduction}

For sports projects, athletic shoes and costume are usually in accordance with the specific requirements of sports design. For example, in the swimming, diving, water polo, water ski, surfing, swimming and other sports, in shoes and costume design, athletes do not drum and pocket water in the underwater action, so that can reduce water resistance; gymnastic shoes and costume should be designed to ensure that athletes technical play freely as the premise, to display their graceful movements of the body; fencing shoes and costume design should first focus on protecting the body, followed by on-demand light. Project design about athletic shoes and costume, combined with ergonomic knowledge, not only can improve game performance, but also can effectively avoid the injured athlete in motion.

This article is skipping example, combining ergonomics knowledge, studying on the effects of ergonomic on athletic shoes and costume design project .This is because skipping simple, physical fitness, and entertain themselves, and has a long history, loved by people of all countries. By studying the impact of skipping athletic performance, combined with ergonomic knowledge, come skipping Contest entries shoes design and solutions.

\section{Ergonomics}

Ergonomics $^{[1]}$ is also called human engineering or ergonomics, which is developed after the Second World War and is a new discipline which is based on human anatomy, physiology and psychology and other features. Understand and grasp the person's work capacity and limits, the appliance, jobs, the environment, living conditions and the human body to adapt disciplines. It is man-machine relationship for the object of study to measurement, statistics, analysis for basic research methods.

Clothing Ergonomics is a discipline which is the study of the relationship between humans and the clothing , focusing on the body's shape and other characteristics of the starting motor skills, give full consideration to the human body and clothing to reconcile and comfort in order to improve the overall clothing and body hungry adaptation skills.

Footwear ergonomics, is through the feet, material and human psychology analysis, so as to satisfy the needs of the wearer of the shoe to maximize.

\section{Ropes skipping sports}

Ropes skipping ${ }^{[2]}$ sports action requirements: when skipping, body need relaxed, eyes straight ahead, shaking the rope to use your wrist, and pay attention not to opening his hands to the sides to ensure 
that the rope passed, off and landing with the toes and forefoot.

Because skipping score bases on the number of calculations, so skipping frequency is a key factor affecting skipping results.So skipping shoes and costume should have good holding fast, continuous elastic and cushioning properties. Another important parameter is the volume and skipping friction. So shoes and costume should be designed to tight, and small shoes is appropriate. We also need minimize the friction, namely fabric to be smooth, the bottom lines of shoes not to be too large.

\section{Research of effect of ergonomics on athletic costume design project}

Competition for skipping sports costume design, combined with ergonomic knowledge, we need to consider the functional aspects from the viewpoint of the fabric, features, aspects, and special sports content and elements of design aspects.

Sportswear fabrics should be dual function of both performance and aesthetics. Thus textile fabrics and technology has become an important factor in determining the trend. Today, through research and the development of new functional fiber fabric dyeing and finishing of the production process and the use of a polymer film and surface processing technology, produce of a beautiful and high-performance sportswear fabrics. Sportswear fabrics often use yarn geometry, tightness, composition and principles of the fabric, to improve the cooling and dehumidification capacity of the fabric. So today, the use of high-tech fabrics will be a necessary condition for improving sports competition clothing professional.

Rope skipping sports from an ergonomic point of view, mainly has the following aspects restricting clothing design criteria: First, the upper sports mainly bases on arm and wrist movement. Exercise time and frequency determine the armpits and back sweat easily, resulting in easy to slide or increased clothing weight of sweat between the skin, thereby affecting athletic performance. Therefore, in the armpits and back breathable mesh fabric design should be used, from the structure of the clothing, the main short-sleeved, the sleeves not too long, not longer than the upper arm to 1/3 appropriate; Secondly, lower limb movement mainly in the lower leg and ankle joints, in order to reduce the resistance movement below the knee, many more shorts are based; In addition, multi-head leaning forward, brow sweat easily, much take sweat scarf or headband to bring fixed.

In the following, we take the study of students in Fujian Province on the fourteenth session of the "Sports Lottery Cup" contest skipping for example. We found, in front of eight in the team scores , men's and women's costume team are shown as Figure1,Figure 2

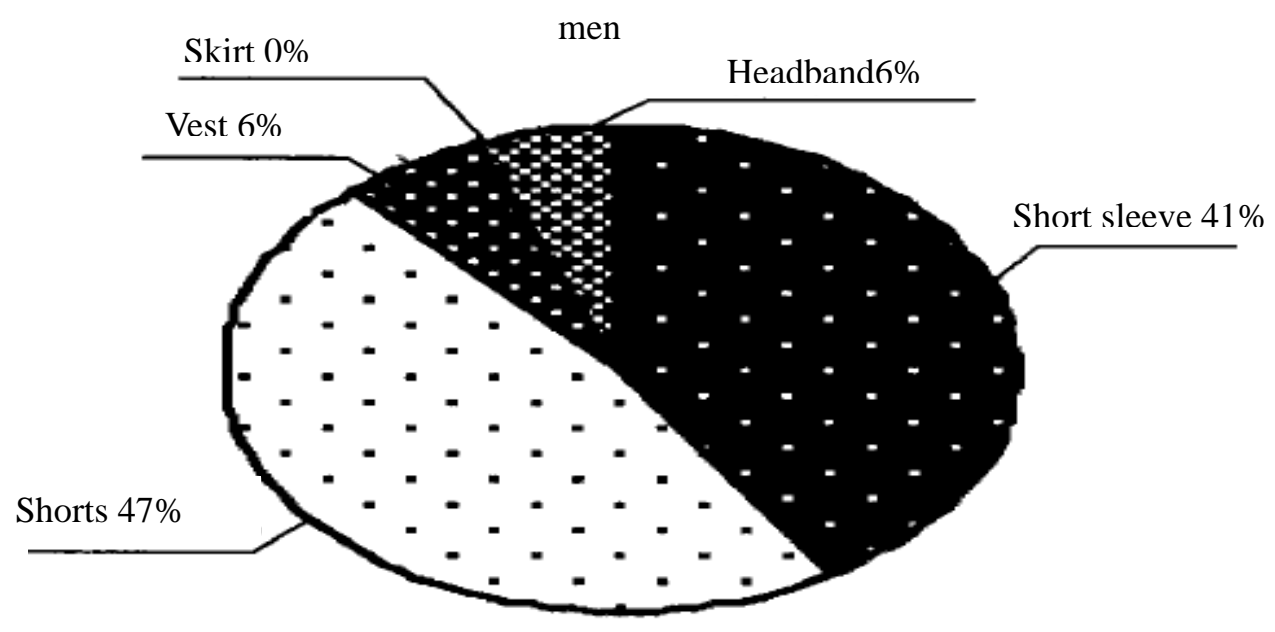

Figure 1 Men's match clothing styles statistics 


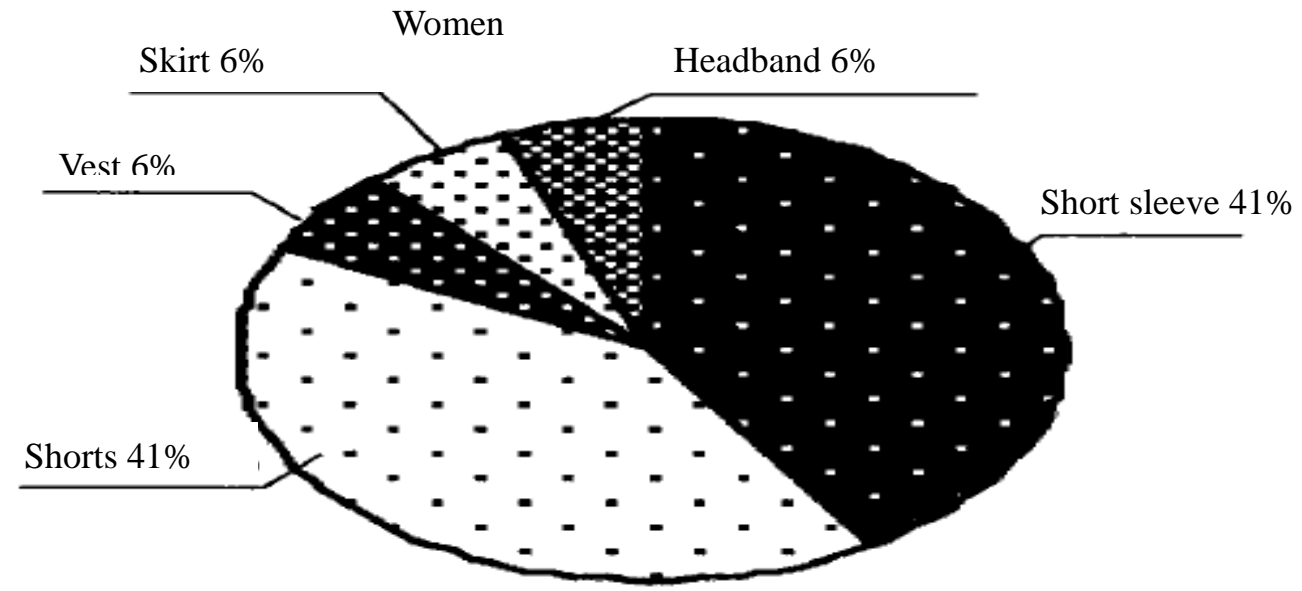

Figure 2 Women's match clothing styles statistics

According to Figure 1,Figure2 we can see that the main sports are short-sleeved or vest style. This classic style is suitable for skipping sports. Competition for skipping sports clothing design, combined with ergonomic knowledge, can meet skipping sports requirement.

\section{Research of effect of ergonomics on athletic shoes design project}

Shoes original function is to protect the feet from injury. With the development of the times, the shoes must be designed to meet the requirements of foot comfort to the maximum extent. That is, the shoes should achieve the most optimal state in physiological structure, psychological needs and changes in other aspects of the movement. Only ergonomics knowledge and shoe production technology combine, we can achieve better goal.

Athletic shoes design based on ergonomic should focus on two aspects: First, to meet the requirements of motor function; the second is to reduce physical exertion of the athletes at the same time, strengthen the protection of bones and tendons.

So skipping footwear design competition program should meet the lightweight and footwear should have good cushioning and flexibility, and the non-slip, wear and flex resistance. Skipping footwear design should make the overall design of the line shape with a reasonable proportion. Color should be out faster.

Selection of skipping shoe uppers with breathable better, should have the folding of material-based. the former should use folding stronger material. The latter should use the strong stability of the material. The heel portion of the skipping shoes should be designed with padded fiberboard, so you can make the shoe better flexibility. Skipping shoe insole portion is provided mainly non-slip, which is generally used to fill the vacancy stereo between insole and footbed feet . Materials commonly uses light and mixed latex or EVA manufacturing regeneration.

Sports shoe soles divided into outsole, midsole and insole. Midsole impact force is mainly used for movement, the material is mainly molded EVA or polyurethane. For the insole, it should have good shock-absorbing function, the material is generally EVA foam rubber. Outsole flex deformation mainly used to bear loads and movement of the body, the material is generally abrasion rubber and MD materials. This design not only help to meet the bouncing, braking, skid, shock absorption and other effects, can also reduce physical exertion, and also play a protective role in the foot bones and tendons.

In the following, we also take the study of students in Fujian Province on the fourteenth session of the "Sports Lottery Cup" contest skipping for example. we study top 8 persons shoes statistical a single project for shake which is shown as Figure 3. 


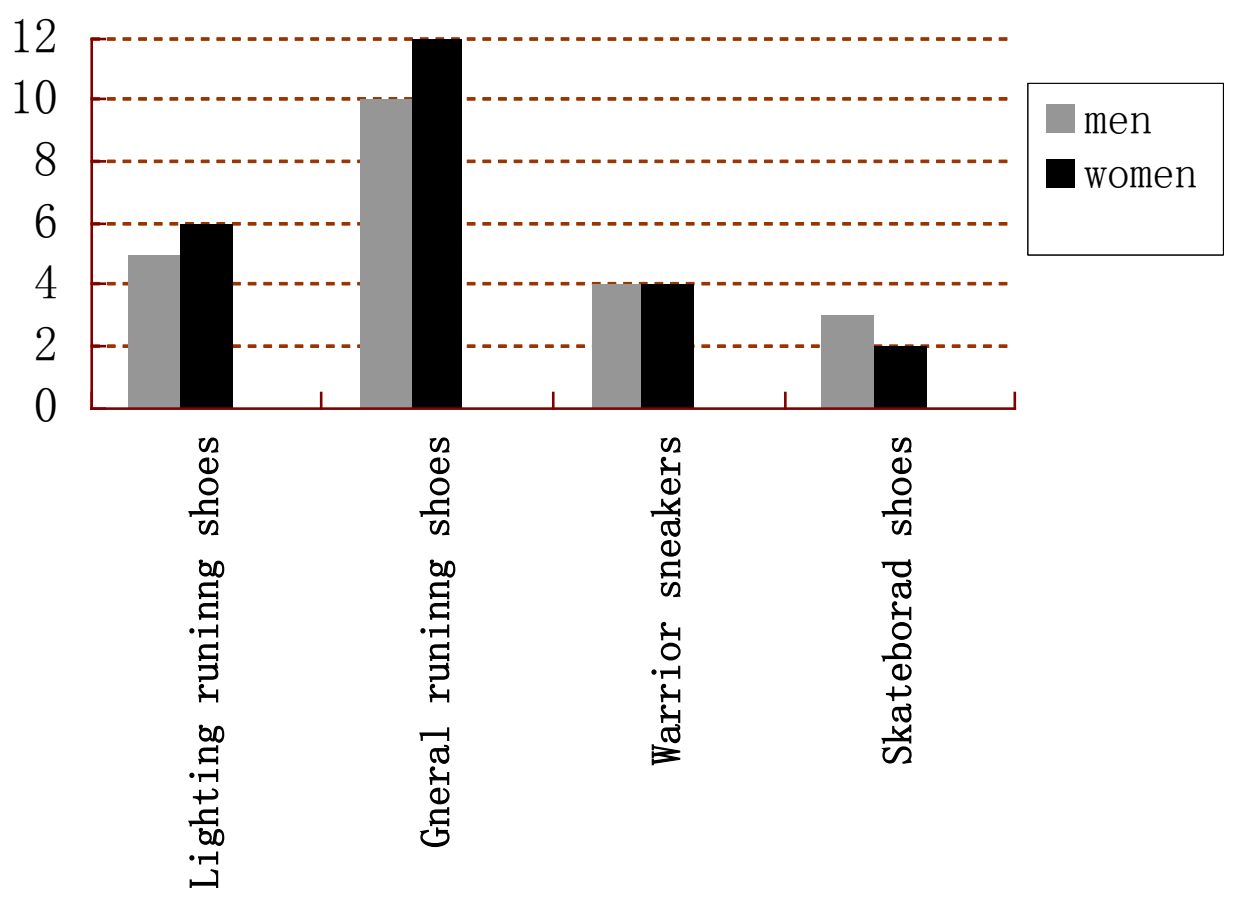

Figure 3 Shoe type game statistics

From Figure 3, the majority of players shoes are running shoes, casual shoes similar mainly. This skipping folding professional sports shoes have good shock absorption and weight reduction particular, which is in line with the needs of sports shoes.

\section{Conclusion}

With the popularity of fitness, sports competition in jumping rope, running and sports competition increasingly fierce, more and more tricks. Combined with ergonomic knowledge, the design of athletic shoes and costume, not only can enhance the athlete's race results, but also can effectively avoid the injured athlete in motion.

Ancient Greece had a famous saying: Man is measure of all things. Athletic shoes and costume design project should be people-oriented. In other words, the rational scientific data, applied to product design, is a manifestation of people - a key factor in the harmony of the product. Only shoes designed with ergonomic sports competition projects shoes really can meet future needs, to be in advantage in the fierce competition.

\section{References}

[1] P.Wang.Ergonomics and its future[J].China Environmental Management College of Technology, 2003,13 (2): 71-73.

[2] J.Li, H.B.Yu.Design of the Shoes and Costume for Ropes Skipping Contest Based on Ergonomics [J]..Quanzhou Normal University.pp:110-112 